Journée européenne contre l'obésité: halte au yo-yo

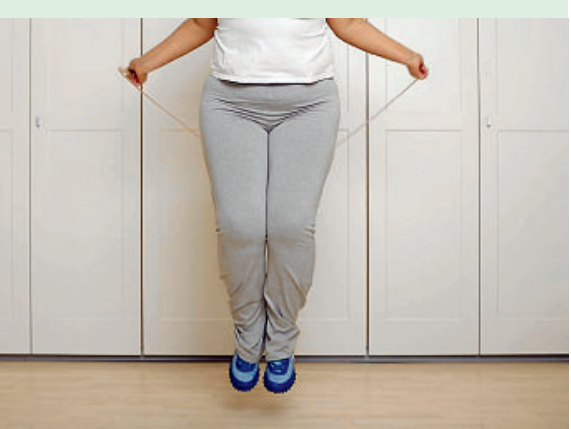

La Journée européenne contre l'obésité est une campagne consacrée à la lutte contre l'obésité organisée chaque année dans toute l'Europe le troisième samedi du mois de mai. L'objectif de la JEO est d'encourager les citoyens européens présentant une surcharge pondérale à mettre en place les changements de style de vie qui s'imposent pour retrouver un poids sain afin d'améliorer leur santé et leur qualité de vie. Car l'obésité est une des premières causes de décès et de maladie qu'il est possible de prévenir en Europe. D'après l'Organisation mondiale de la Santé (OMS), la surcharge pondérale et l'obésité sont chaque année à l'origine de plus d'un million de décès et de 12 millions d'années de vie en mauvaise santé.

(JEO)

\section{Mehr Aidsfachleute} für Simbabwe

Das Ausbildungszentrum, das die Newlands Clinic dank einer grosszügigen Spende der Bernhart-Matter-Stiftung in Simbabwes Hauptstadt Harare eröffnen konnte, hat seinen Betrieb erfolgreich aufgenommen. Dank dem neuen Zentrum der Stiftung Swiss Aids Care International von Prof. Ruedi Lüthy können deutlich mehr einheimische Ärzte und Pflegepersonen im Umgang mit der Krankheit Aids ausgebildet werden als bisher. Pro Jahr absolvieren mehr als 200 Personen die Kurse und wenden ihr Wissen anschliessend in Kliniken in ganz Simbabwe an.

(Swiss Aids Care International)

\section{Gefässtachometer in der Testphase}

Forscher der Berliner Charité, des Gefässzentrums Berlin und des Max-Delbrück-Zentrums haben ein Verfahren entdeckt, das mit Ultraschall und einem Zusatzgerät erstmals messen kann, wie schnell das Blut in der Arterie maximal beschleunigt und entschleunigt wird. Das Gerät könnte helfen, den Zustand der Durchblutung bei Risikopersonen genauer und einfacher darzustellen. Der Gefässtachometer misst die Duchblutung mit einer Ultraschallsonde von aussen an der Halsschlagader, im Bereich des Ohres, in der Leiste oder im Kniebereich. Damit können Gefässerkrankungen möglicherweise schon früher entdeckt werden, ohne dass Patienten eine aufwendigere Farbdoppleruntersuchung oder Angiographie mit Kontrastmittel benötigen. Zurzeit wird das Gerät in einer Studie geprüft.

\section{(Deutsche Gesellschaft für Angiologie)}

\section{Diabetes schränkt Berufsleben kaum ein}

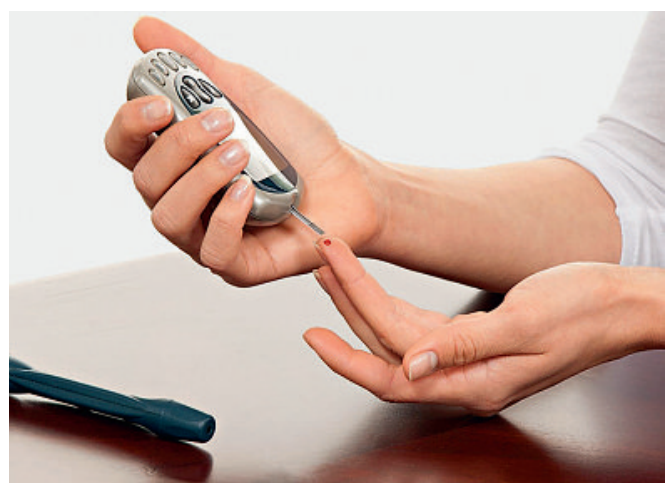

Häufiges Blutzucker-Messen am Arbeitsplatz stösst bei informierten Arbeitskollegen auf viel Verständnis.

\section{Plus de responsabilisation pour la santé des femmes et des enfants}

La Commission de l'information et de la responsabilisation en matière de santé de la femme et de

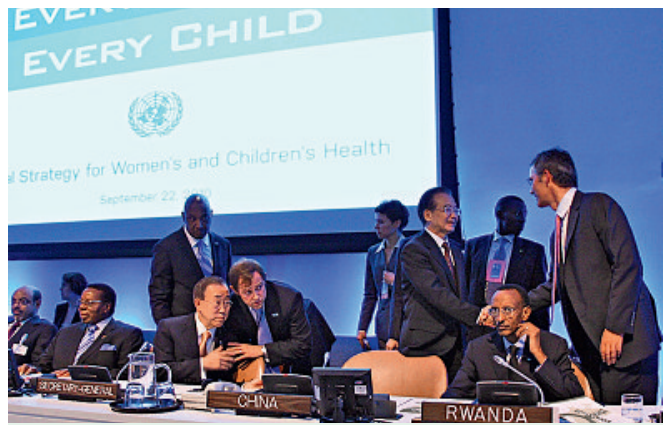

La Commission de l'information et de la responsabilisation en matière de santé de la femme et de l'enfant des Nations Unies a approuvé de nouvelles recommandations.

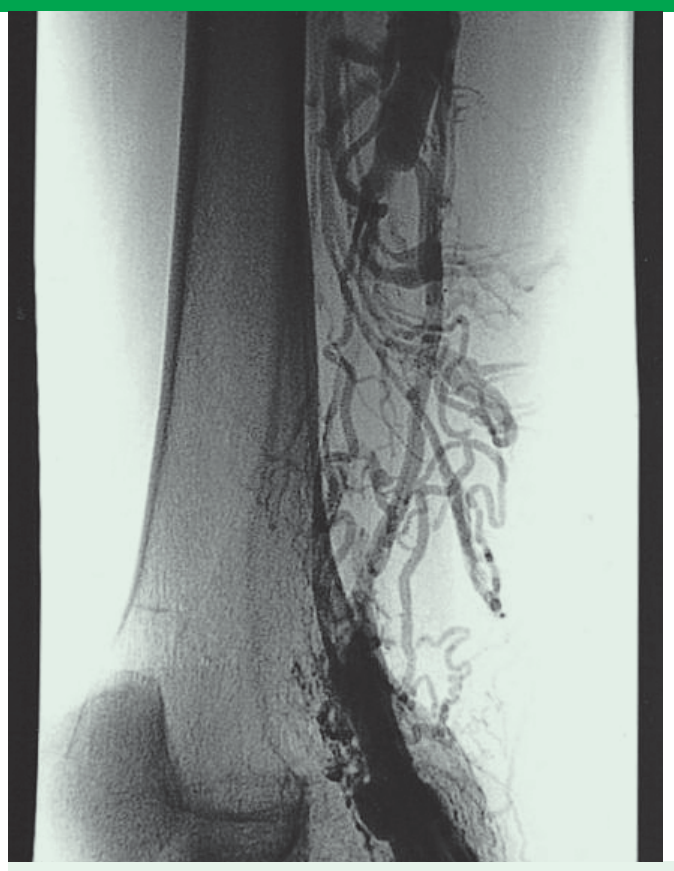

Ob ein neues Messgerät aufwendige Angiographien (hier eine tiefe Beinvenenthrombose) ersetzen kann, untersucht eine Studie der Berliner Charité.

Schulabgänger mit Diabetes Typ 1 sind oft unsicher, ob sie den Wunschberuf ergreifen dürfen. Und Berufstätige mit Diabetes Typ 2 halten ihre Krankheit am Arbeitsplatz geheim. Dabei macht Diabetes Typ 1 oder 2 nur selten eine Versetzung oder Umschulung nötig. Darauf hat diabetesDE anlässlich des Welttages für Sicherheit und Gesundheit am Arbeitsplatz am 28. April hingewiesen. Das Wissen der Arbeitskollegen um die Erkrankung fördert das Verständnis z.B. für Blutzucker-Messungen. Auch für Notfälle ist ein gutinformiertes Umfeld vorteilhaft. Nur für die Ausübung von Berufen, bei denen Diabetiker wegen Unterzuckerung sich selbst und andere gefährden könnten, etwa Pilot oder Busfahrer, gilt die Erkrankung als Hinderungsgrund.

(diabetesDE)

l'enfant des Nations Unies a approuvé de nouvelles recommandations appelant à un niveau de responsabilisation sans précédent pour sauver plus de vies de femmes et d'enfants dans les pays en développement. Les dix recommandations prévoient des approches spécifiques pour aider les pays à élaborer de meilleures méthodes de collecte de données sanitaires importantes pour mieux comprendre les besoins en matière de santé et savoir où concentrer les ressources, de mettre sur pied un système coordonné pour le suivi des dépenses de santé concernant les femmes et les enfants et d'assurer une surveillance nationale et mondiale. Le rapport final de la Commission sera soumis au Secrétaire général de l'ONU, M. Ban Ki-moon. Pour plus d'informations: www.everywomaneverychild.org

$(\mathrm{ONU})$ 